\title{
Immune Neuroplasticity (Power Within) is Weakened by Vaccines and Drugs (Power Without): Mitochondrial Sink Holes, Genomic Destabilization and Immune Disorders
}

\author{
Hypothesis \\ Mahin Khatami, Retired, National Cancer Institute, National Institutes of Health, \\ Bethesda, MD 20892, USA; Correspondence: mkgoodness@aol.com
}

\begin{abstract}
A parallel between defense powers of sovereign nations and effective immunity that guards health is relevant to demonstrate vulnerability of immune system under external forces (vaccines, drugs). History demonstrated that sovereignty (power within) of small nations often threatened or destroyed by military might of powerful nations (power without) who use false-flags and propaganda for motives that are financial-control-driven. Similarly, we propose that body's complex immune neuroplasticity (power within, adaptive, horizontal) is stretched-thin and weakened by the external forces, particularly by vaccination of the unborn/newborn or immunecompromised individuals. Validity of genomics (innate, perpendicular) as origins of 'hereditary' diseases (eg, allergies, diabetes, cancers) that for a century dominated research and treatment is also challenged.
\end{abstract}

In conclusion, we propose that the pressure/power from within creates life with potential to sustain health, while the pressure/power from without, weaken and destroy life.

Keywords: Acute and Chronic Inflammation; Bioenergetics; Constituent and Inducible Receptors; Fetus; Genomics; Glycolysis; Immunity; Immune compromised; Immune disorders; Infant; Inheritance; Mitochondrial; Newborn; Placenta; Power within; Power without; Sovereignty; Throphoblast; Tumorigenesis; Tumoricidal; Vaccines; Yin-Yang

\section{Introduction:}

1. Immune Neuroplasticity (Power Within): Bioenergetics Requirements at Different Stages of Life: Vulnerability toward External Powers (Vaccines).

If there is no enemy within, the enemy without can do us no harm. Sir Winston Churchill

Effective immunity is referred to an amazingly complex bio-electromagnetic crosstalk between immune and non-immune (vascular-hormonal-neuronal-metabolicgenomics-lipidomics) systems, collectively described as cell-mediated and humoral immunity (CMI/HI). Time-energy-dependent interactions (negative and positive signal switches) of autonomic sympathetic and parasympathetic properties of immunity possess high degrees of molecular intelligence for protecting health. We defined effective immunity as the balance between 2 highly regulated and biologically opposing arms, Yin (tumoricidal, degeneration, tear) and Yang (tumorigenic, regeneration, wear) properties of self-terminating acute inflammation (immune surveillance) [1-6]. 
Within two years after birth and exposure to air oxygen and environmental conditions, mitochondria, organ functionalities and tumoricidal (Yin) arm of effective immunity (power within) are completed [1,2].

Optimal operation of immunity (polarization-depolarization capacity of Yin-Yang) requires differential bioenergetics (biorhythms) for defending the body against exogenous or endogenous threats with the following features [1-6]:

(a). Yin events demand high energy expenditure (ATP hydrolysis) from mitochondrial oxidative phosphorylation to destroy foreign entities and injured tissues. Stimuli-induced Yin signals include specific danger molecules, receptors, vasoactive and cytotoxic/neurotoxic agents, cytokines/ chemokines (eg, TLRs, ILs, TNF-a, histamine, ILs, oxidases).

(b). Yang events require low energy from cytoplasmic glycolysis to neutralize and repair or remodel tissue, during which time mitochondria shut down for regeneration of TCA intermediates. Yang processes express wound healing mediators (eg, TNFdRs, Ils, IRAK-M, SODs, GFs, catalase, IFN, hormones, kinases).

(c). Acute inflammation differentially affects tissues that are immune-responsive (e.g., epithelium, mucus-secreting cells, vasculature) or immune-privileged (eg, CNS, $\mathrm{BBB}$, neuroretina, reproductive system).

At different stages of life, fundamental pathways that profoundly influence activities of mitochondria and immunity (tissue growth and necrosis) include pathways that are involved in constituent and inducible receptors (pattern recognition molecules) and related enzymes [eg, pyruvate kinases (PKM1, for muscle, heart or brain function; PKM2 (embryonic tissues); PKL (liver), PKR (erythrocyte)] or diverse insulin Rs; ARNT-HIF1; histidine-histamine $\mathrm{Rs}$ and numerous other receptors that contribute to posttranslational regulation, architectural integrities and function of tissues (eg, proton pumps, cation-anion transporters, water channels, surface molecules, growth or apoptotic factors). For example, numerous receptor molecules contribute to visual transduction, bone and lipid biosynthesis, bioenergetics, cellular infiltration, differentiation and growth, nuclear/chromosomal or chromatin activities, neuronal pathways and pattern recognition of pathogens or foreign elements and are involved in tissue necrosis (degeneration) or growth (regeneration) [1-10].

After birth the genetically-determined constituent receptor molecules are often immediately influenced by the signals they receive from the environment and adapt to the quality of nutrition (initiated from mother's milk) and exposures to a variety of bioactive agents, microorganisms, environmental chemical and biological hazards, including pathogen-specific vaccines, shapping body's immune neuroplasticity (horizontal, adaptive) [1-6, (manuscript in preparation)].

\section{Vaccine Toxicities: Cause, Exacerbation and Consequence of Mild, Moderate or Severe Immune Disorders: Weakening Effective Immunity (Power Within):}

Despite lack of systematic studies on vaccines development and existence of major knowledge gaps or inconclusive data on casual relationships between vaccines and immune disorders, an overview of information on vaccination of unborn/newborn/infant and immune-compromised individuals or comparison of vaccinated and unvaccinated 
group provide sufficient evidence that current pathogen-specific vaccines are likely the cause and consequence of significant increased in immune disorders in four generations in America (Figure 1)[1,2,4,11-14].

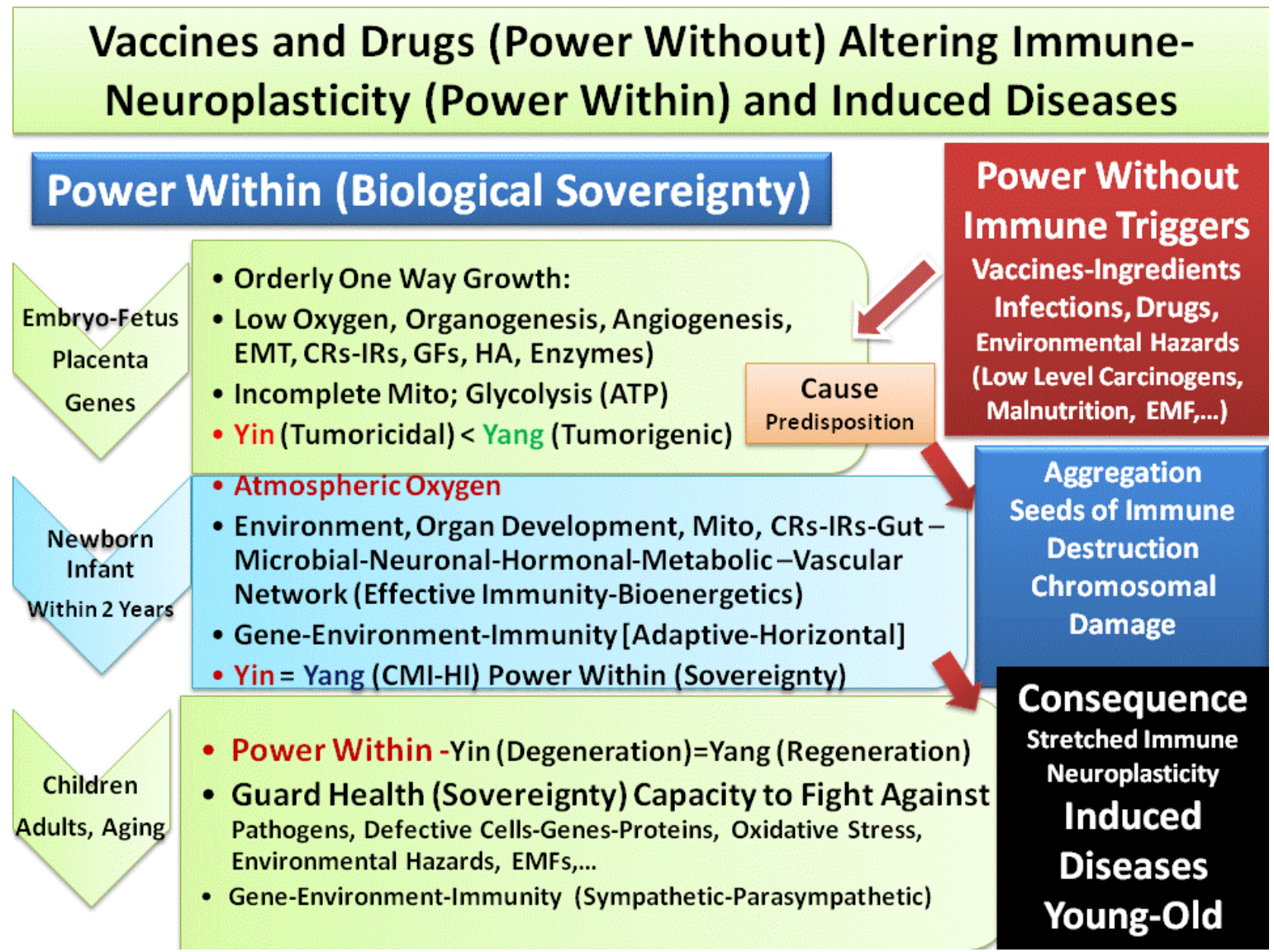

Figure 1. Schematic representation of immune neuroplasticity (power within, biological sovereignty) at different stages of life. It depicts that fetus organogenesis and angiogenesis occur under hypoxia where glycolysis is the main source of energy for unidirectional growth (Yang) within protected environment of placenta. Mitochondria and tumoricidal (Yin) arm of immunity are not completely developed during fetus growth. Power within (effective immunity) and completion of organ development and immunity (gene-environment-immunity, balance between Yin and Yang) are depicted to be completed within 2 years after birth and exposure to atmospheric oxygen. Expression of various constituent or inducible receptors (CRs, IRs), growth factors (GFs), epithelial/mesenchymal transition (EMT), are shown to be influenced by environmental factors (power without) at different stages of life. Immune triggers such as vaccines and ingredients, infections, low level carcinogens are depicted as seeds of immune destruction affecting mitochondrial bioenergetics and immunity that alter chromosomal/genomics stability; bases for cause, exacerbation (aggregation) or consequence of induced diseases. See Text.

Vaccination of pregnant women, at different stages of embryo-fetus growth could disturb and destabilize orderly organogenesis and angiogenesis and immune 
neuroplasticity, immediately after birth or weeks, months or years later. Vaccination of unborn could alter delicate biology of trophoblast (asexual)-embryo-fetus-placenta and epithelial-mesenchymal transition and the timely expression or inhibition of constituent or inducible receptors and mitochondrial development that are required for maintenance of health throughout life [1,2].

Vaccine-associated injuries (eg, fatigue, autism, allergies, hypotonia, neuropathological episodes of epilepsy, Rett syndrome or encephalomyopathy, autoimmune and neurodegenerative disorders or cancers) are likely the results of retardation of one or more molecular defects in immune response profiles. Vaccine ingredients [eg, aluminum, thimerosal, detergents, glyphosate, engineered DNA/RNA, yeast extracts, aborted fetus tissues, L-His, hydrolyzed gelatin or filterable viruses] could retard/compete with electrochemical properties of proteins that require trace elements (eg, $\mathrm{Fe}^{+2,+3}, \mathrm{Cu}^{+1,+2}, \mathrm{Zn}^{+2}, \mathrm{Mg}^{+2}$ ) for their functions [eg, inner/outer mitochondrial membrane complexes (I, II, III or IV), metal carriers, enzymes]. Mitophagy is associated with impaired chromosomal-genetic activities and mismatched expression or coexpression of pro-, anti-inflammatory mediators, immunoglobulins, surface molecules, memory complexes of B/plasma and adaptive regulatory $\mathrm{T}$ cells (Treg), altered Th1/Th2 ratios, GSH: GSSH, NAD ${ }^{+}$: NADH recycling and altered tissue oxido-redox potentials. Unborn/newborn vaccination could also profoundly influence composition and behaviors of microbiomes in gastrointestinal tract (gut-brain axis) and neurophysiology of histidinehistamine pathways often associated with altered genetic/chromosomal stability and epigenetic modifications [1,2,4,10-13].

It is noteworthy, that stimuli (vaccines)-induced allergies are generally classified as clinical symptoms of three types of tissue responses a) IgE-mediated (acute, type 1 reactions); b) non-IgE-mediated (cellular or delayed type hypersensitivity); and c) mixed (IgE-mediated and delayed type responses)] and bases for a wide range of chronic disorders $[1,2,4,9,10]$.

In brief, tissue overstimulation by vaccine and impaired mitochondrial function is likely to retard/weaken the fighting capacity of immunity (power within). Overvaccination would stretch-out/thin the natural polarization-depolarization capacity of Yin-Yang events that are features of unresolved inflammation (oxidative stress) that would lead to immune tolerance or intolerance. Unresolved inflammation (subclinical, oxidative stress) was demonstrated as common denominator in induction of nearly all acute and chronic diseases.

3. Immune-Neuroplasticity (Adaptive, Horizontal) v. Genomic (Innate, Perpendicular): Challenging Validity of Inherited Diseases.

Different theories of aging biology (eg, oxidative stress, genomics, telomere, immunity) are reviewed elsewhere [2]. Below, we challenge the validity of a century-old theory of inheritance-genetics-somatic mutations that dominated heavy investment that attempted to describe the origins of many diseases (eg, sickle cell anemia, progeria, allergies, cancers) $[1,2,4,15,16]$ :

Cancer and many neurodegenerative and autoimmune diseases became inducible 'inherited' diseases in the last four generations; or ever since the public consumed viruscontaminated polio vaccines in 1955's/1960's; 
(a). Nearly all known classic disease categories (congenital, inherited, neonatal or induced) that occurred at the rates of $1-5 \%$, in last century, shifted to increase induced diseases (rates of 30-50\%) in young and old, by heavy consumption of drugs and overvaccination or exposures to low level carcinogens;

(b). After birth, inducible signals that contribute to gene-environment-immune interactions possess adaptive behaviors with super-packages of electrochemical behaviors that parallel neuronal brain function;

(c). Sympathetic -parasympathetic responses that shape immunity present horizontal (adaptive, stretchable) behaviors that cannot be explained by limited nature of genomics (innate, perpendicular) to define inherited diseases (e.g., cancers);

The author proposes that conditions that traditionally are identified as inherited diseases are the results of inflammation-induced irreversible damage to parental-ancestral chromosomes that permanently alter expression of constituent and adaptive receptors and tissue functions. 'Inherited' disorders [eg, allergies, emphysema, diabetes and cardiovascular complications, neurodegenerative and autoimmune diseases (eg, autism, Alzheimer's, lupus, ALS), cancers] are likely the results of altered immune responses that secondarily destabilize chromosomal activities (eg, DNA/RNA repairs mechanisms, epigenetic modifications and related protein expression profiles).

Support for this concept comes from analyses of our original studies along with integration of data on developmental biology, genetics of infectious diseases, vaccine injuries and the current disease-status in young and old in America [1-6,11-14]. Our models of ocular inflammatory diseases resulted in a series of time-dependent developmental phases of immune dysfunction toward multistep tumorigenesis and angiogenesis. Hypersensitivity reactions that developed in the newborn, born from highly sensitized animals suggested pre-genetic disposition. Data on vaccination of unborn/newborn or individuals who are immune-suppressed reveal the likelihood of alterations of time-energy-dependent immune responses that would secondarily damage stabilities of chromosomal components and alter expression or co-expression of pro-, and anti-inflammatory responses toward disease induction $[1,2,9]$.

\section{Concluding Remarks: Promoting Power Within for Improving Health:}

The initial immunity and tolerance in respiratory and gastrointestinal tracts develop during fetus organogenesis and angiogenesis in the protective environment of placenta. Unborn or newborn exposures to even 'safe' vaccines would_deny the defense capacity of body before immunity is completed. Current vaccines threaten mitochondrial bioenergetics and proper expression of constituent and inducible receptors that are required for anabolism-catabolism processes, and are the likely bases in induction short-, or long-term health problems. Universal safe vaccines or prophylactics that prevent diseases should mimic the natural course of immunity that would enhance the biological power from within. Efforts to limit excessive activation of gene-environment-immune neuroplasticity are keys for maintaining balance in Yin and Yang capacity of effective immunity.

It is so recognized that the pressure/power from within creates life with potential to sustain health, while the pressure/power from without, weaken and destroy life.

Funding: This research received no external funding. 
Data Availability Statement: The study did not report any data.

Conflicts of Interest: The author declares no conflict of interest.

\section{References:}

1. Khatami M (2020) Deceptology in cancer and vaccine sciences: Seeds of immune destruction, mini electric shocks in mitochondria- Alter neuroplasticity/electrobiology of response profiles and increased diseases in 4 generations. A hypothesis. Clin Trans Med. First published: 19 December 2020 https://doi.org/10.1002/ctm2.215

2. Khatami M (2017) In Inflammation, aging and cancer: biological injustices to molecular village that guard health. Springer, Berlin, pp 1-389.

http://www.springer.com/gp/book/9783319664736

3. Khatami M (2008) "Yin and Yang" in inflammation: duality in innate immune cell function and tumorigenesis. Exp Opin Biol Ther. 8: 1461- 1472.

4. Khatami M (2018) Cancer; an induced disease of twentieth century! Induction of tolerance, increased entropy and 'Dark Energy': loss of biorhythms (Anabolism v. Catabolism). Clin Transl Med. 2;7(1):20. doi: 10.1186/s40169-018-0193-6.

5. Khatami M (2016) Is cancer a severe delayed hypersensitivity reaction and histamine a blueprint? Clin Transl Med. 5:35. https://doi.org/10.1186/s40169-016-0108$\underline{3}$

6. Khatami M (2011) Unresolved inflammation: 'immune tsunami' or erosion of integrity in immune-privileged and immune-responsive tissues and acute and chronic inflammatory diseases or cancer. Exp Opin Biol Ther. 11: 1419- 1432

7. Raefsky SM, Mattson MP (2017) Adaptive responses of neuronal mitochondria to bioenergetic challenges: Roles in neuroplasticity and disease resistance. Free Radic Biol Med. 102:203-216. doi:10.1016/j.

8. Kallio PJ, Pongratz I, Gradin K, McGuire J, Poellinger L (1997) Activation of hypoxia-inducible factor 1alpha: posttranscriptional regulation and conformational change by recruitment of the Arnt transcription factor. Proc Natl Acad Sci U S A. 94(11):5667-72. doi: 10.1073/pnas.94.11.5667. PMID: 9159130; PMCID: PMC20836.

9. Khatami M. Chronic inflammation: synergistic interactions of recruiting macrophages (TAMs) eosinophils (Eos) with host mast cells (MCs) and tumorigenesis in CALTs. MCSF, suitable biomarker for cancer diagnosis! Cancers. 2014;6:297-322.

10. Khatami M (2009) Inflammation, aging, and cancer: tumoricidal versus tumorigenesis of immunity: a common denominator mapping chronic diseases. Cell Biochem Biophys. 55:55-79.

11. Graf WD, Marin-Garcia J, Gao HG, et al (2020) Autism associated with the mitochondrial DNA G8363A transfer RNA(Lys) mutation. J Child Neurol;15:357-361.

12. Giannotta G, Giannotta N (2018) Vaccines and Neuroinflammation. Int J Pub Health Safe. 3;3.

13. Khatami M (2016) Safety concerns and hidden agenda behind HPV vaccines: another generation of drug-dependent society? Clin Transl Med. 5(1):46

14. Cowan $T$ (2018) In: Vaccines,Autoimmunity, and the ChangingNature of Childhood Illnesses. White River Junction,Vermont, London, UK: Chelsea Green Publishing; 1-169. 
15. Pairo-Castineira, E., Clohisey, S., Klaric, L. et al. (2020) Genetic mechanisms of critical illness in Covid-19. Nature . https://doi.org/10.1038/s41586-020-03065-y

16. Sayaman RW, Saad M, Thorsson V, Hu D, Hendrickx W, Roelands et al (2021) Germline genetic contribution to the immune landscape of cancer. Immunity. 54(2):367386.e8. doi: 10.1016/j.immuni.2021.01.011. PMID: 33567262. 